Historic, Archive Document

Do not assume content reflects current scientific knowledge, policies, or practices. 



\section{SPECIAL WHOLESALE PRICE LIST}

\section{For Market Gardeners and Large Planters}

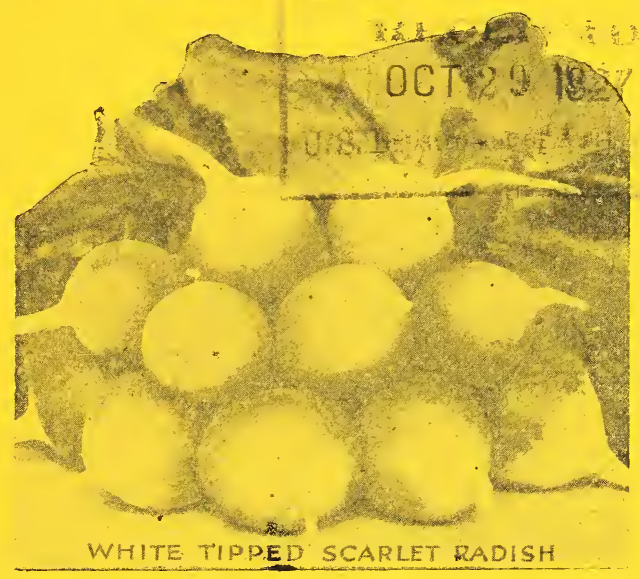

\section{T. Lee Adams Seed Co. SEEDSMEN}

Kansas City, Mo.

PHONE VICTOR 8404

\section{GUARANTEE}

We buy and sell THE BEST SEEDS THAT GROW. But it is impractical to guarantee seeds, as the best seeds will fail under improper treatment. Some may be sown in too dry ground or too wet, too deep or too shallow. Some hardy seeds stand cold weather and will grow where the soil is too cold for others and would cause them to decay. Insects may attack and destroy them. For these reasons we give no warranty, express or implied, as to description, quality, or any other matter of any seeds, bulbs or plants we send out and we will not be in any way responsible for the crops. If the pur. chaser does not accept the goods on these terms, they are at once to be returned.

A pleased customer brings us two new ones.

We thank you for your liberal patronage and kind expressions and will always strive to merit your continued favors.

Wishing you a prosperous year, we remain,

Yours very truly, T. LEE ADAMS SEED CO.

Without good seeds it is impossible to produce good crops. Our aim is to supply only the highest possible quality produced. You cannot buy better seeds no matter what you pay.

Field seed prices are all subject to change without notice. Write for samples and special prices on your field seed requirements. We will save you money. 


\section{HOW TO ORDER}

Please sign your name, post office, county and state on every letter sent us. You may remit at our risk by Post. Office Money Order, Check, Draft, Express Order, Cash, Stamps or by Registered Letter. We guarantee safe and prompt delivery whether sent by mail, express or freight.

\section{HOW SEEDS ARE SHIPPED}

Prices quoted in this List include transportation charges except on potatoes, field seeds, feed, berry boxes, baskets, plants, insecticides, fertilizers and implements, which will be shipped at buyer's expense.

In ordering please mention whether wanted by mail, express or freight.

Berry Boxes and Fruit Baskets at Market Prices

\section{TERMS}

Cash with all orders. We make no charge for boxes, packing or drayage. Bags at market price. All goods delivered to transportation company free of charge.

All prices quoted in this list are subject to market fluctuations or prior sale.

Peas, Beans, Sugar Corn, Potatoes, field seeds, onion sets net-no discount.

Discounts-On large orders of vegetable seeds we offer the following rates of discount for cash:

$\$ 10.00$ and under $\$ 20.00 \ldots \ldots \ldots 2$ per cent

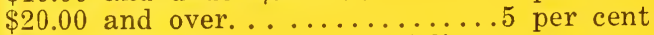

We guarantee safe and prompt delivery on every order.

\section{ASPARAGUS}

One-half Pound at Pound Price Oz

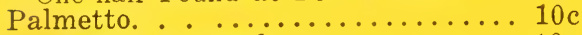

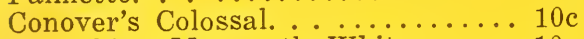

Columbian Mammoth White...... 10c

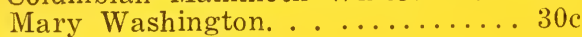

$\begin{array}{lr}1 / 4 \text { Lb. } & \text { Lh. } \\ 25 c & 75 c \\ 25 c & 75 c \\ 25 c & 75 c \\ 9 \cup c & \$ 3.00\end{array}$

\section{BEANS, DWARF OR BUSH-Green Podded}

25 Pounds at 100 Pound Price Lb. Early Bountiful. . ...... 35c Burpee's Stringless Greenpod 35c Giant Stringless Greenpod.. 35c Longfellow. .......... 35c Black Valentine. ........ 35c Red Valentine. ........ 35c Full Measure. .......... 35c

$\begin{array}{rrr}5 \text { Lbs. } & 10 \mathrm{Lbs} . & 100 \mathrm{Lbs} . \\ \$ 1.25 & \$ 2.25 & \$ 18.00 \\ 1.25 & 2.25 & 18.00 \\ 1.25 & 2.25 & 18.00 \\ 1.25 & 2.25 & 18.00 \\ 1.25 & 2.25 & 18.00 \\ 1.25 & 2.25 & 18.00 \\ 1.25 & 2.25 & 18.00\end{array}$

\section{BEST DWARF YELLOW PODDED OR WAX}

25 Pounds at 100 Pound Price Lb. Currie's Rust Proof Wax... 35c Challenge Black Wax..... 35c Prolific German Black Wax 35c Pencil Pod Wax.................. Golden Wax. ...........35c Wardwell's Kidney Wax... 35c Dwarf Unrivaled Wax..... 35c Webber's Wax. ........ 35c

$\begin{array}{rrr}5 \text { Lbs. } & 10 \text { Lus. } & 100 \text { Lbs. } \\ \$ 1.40 & \$ 2.50 & \$ 20.00 \\ 1.40 & 2.50 & 20.00 \\ 1.40 & 2.50 & 20.00 \\ 1.40 & 2.50 & 20.00 \\ 1.25 & 2.25 & 18.00 \\ 1.40 & 2.50 & 20.00 \\ 1.40 & 2.50 & 20.00 \\ 1.40 & 2.50 & 20.00\end{array}$

\section{BEANS, DWARF LIMA}

25 Pounds at 100 Pound Price Lb. Henderson's Bush Lima.... 40c Fordhook Bush Lima..... 45c Burpee's Bush Lima....... 45 Dreer's Bush Lima........ 45c

$\begin{array}{rrr}5 \text { Lbs. } & 10 \text { Lbs. } & 100 \text { Lbs. } \\ \$ 1.40 & \$ 2.50 & \$ 20.00 \\ 1.75 & 3.25 & 27.00 \\ 1.75 & 3.25 & 27.00 \\ 1.75 & 3.25 & 27.00\end{array}$

5 Lbs. 10 Lbs. 100 Lbs. $\begin{array}{lll}1.75 & 3.25 & 27.00\end{array}$ $\begin{array}{lll}1.75 & 3.25 & 27.00\end{array}$ 27.00 
BEST VARIETIES POLE BEANS

25 Pounds at 100 Pound Price Lb. 5 Lbs. 10 Lbs. 100 Lhs.

Kentucky Wonder or Old

Homestead. ......... 35c \$1.25 \$2.25 $\$ 18.00$

Kentucky Wonder Wax...4 40c $\quad 1.50 \quad 2.75 \quad 24.00$

White Kentucky Wonder... 35c $\quad 1.65 \quad 3.00 \quad 25.00$

Red Speckled Cut Short.... 35c $\quad 1.40 \quad 2.50 \quad 20.00$

Lazy Wife. ...........4 40 $\quad 1.50 \quad 2.75 \quad 22.00$

King of the Garden Lima. 40 $\quad \begin{array}{lll}1.65 & 3.00 & 25.00\end{array}$

Large White Lima. . . . . . 40 40 $\quad 1.65 \quad 3.00 \quad 25.00$

Small White Lima, Carolina

or Sieva. . ........4 40c $1.65 \quad 3.00 \quad 25.00$

\section{BEETS-For Table}

Five Lbs. and Over 5c Less Per Lb.

One-half Pound at Pound Price

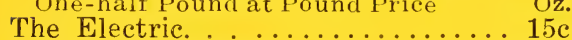

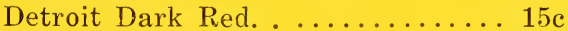

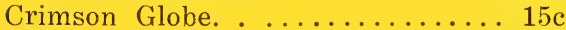

Eclipse. ................ 15c

Crosby's Egyptian. . ......... 15c

Early Blood Turnip. .......... 15c

Early Wonder.............. 15c

Dewing's Improved. . . . . . . . . . . . . . . . . .

Half Long Blood. . .......... 15c

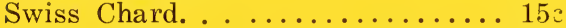

Nutting's Gem. . . . . . . . . . . 15c

$\begin{array}{rr}1 / 4 \mathrm{Lb} & \text { Lb. } \\ 25 \mathrm{c} & 75 \mathrm{c} \\ 25 \mathrm{c} & 75 \mathrm{c} \\ 25 \mathrm{c} & 75 \mathrm{c} \\ 25 \mathrm{c} & 75 \mathrm{c} \\ 25 \mathrm{c} & 75 \mathrm{c} \\ 25 \mathrm{c} & 75 \mathrm{c} \\ 35 \mathrm{c} & \$ 1.00 \\ 25 \mathrm{c} & 75 \mathrm{c} \\ 25 \mathrm{c} & 75 \mathrm{c} \\ 25 \mathrm{c} & 75 \mathrm{c} \\ 35 \mathrm{c} & \$ 1.00\end{array}$

\section{BEETS-Mangel and Sugar}

Five Lbs. and Over 5 c Less Per Lb.

One-half Pound at Pound Price Oz.

$1 / 4 \mathrm{Lb} . \quad \mathrm{Lb}$.

Golden Tankard. ............... 10c

Vilmorine's Sugar. ........... 10c

Giant Half Sugar White......... 10c

$20 \mathrm{c} \quad 50 \mathrm{c}$

$20 \mathrm{c} \quad 50 \mathrm{c}$

$20 \mathrm{c} \quad 50 \mathrm{c}$

$20 \mathrm{c} \quad 50 \mathrm{c}$

CABBAGE

One-half Pound at Pound Price Oz.

Golden Acre.............. 75c

$1 / 4$ L.b. Lb.

$\$ 2.75 \$ 10.00$

$1.50 \quad 4.50$

Copenhagen Market Imported.... 50

$1.25 \quad 3.50$

$\begin{array}{lll}\text { Early Jersey Wakefield. ........ 35c } & 1.00 & 3.00 \\ \text { Charleston Large Wakefield...... 35c } & 1.00 & 3.00\end{array}$

Glory of Enkhuizen............50c $1.50 \quad 4.50$

Cannon Ball (Late). ..........50 $\quad 1.50 \quad 5.00$

Early Dwarf Flat Dutch......... 35c $1.00 \quad 3.00$

Henderson's Succession. . ........35c $1.00 \quad 3.00$

Burpee's Allhead. . ............... 35c $1.00 \quad 3.00$

Henderson's Early Summer....... 35c $1.00 \quad 3.00$

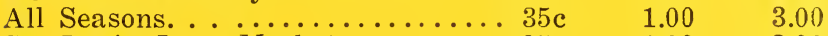

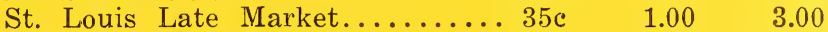

Premium Late Flat Dutch.........35c $1.00 \quad 3.00$

Danish Ballhead, Short Stem..... 40c $\quad 1.25 \quad 4.00$

Chinese Cabbage (Wong-Bok)....50 50 $\quad 1.50 \quad 5.00$

Sure Head. .................35c $1.00 \quad 3.00$

Mammoth Red Rock. ...........35c $1.00 \quad 3.00$

American Perfection Drumhead

Savoy. ........................

$1.00 \quad 3.00$

CARROTS

Five Lbs. and Over 5c Less Per Lb.

One-half Pound at Pound Price Oz.

Early Scarlet Horn. . ......... 15c

Chantenay. . ............ 15c

Extra Early Half Long Nantes... 15c

Danver's Half Long. . . . . . . . . . 15c

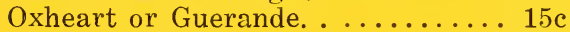

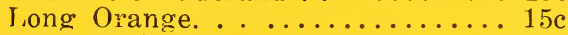

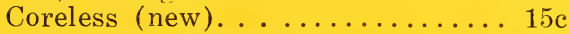

Long White Belgian. ......... 15c

Long Yellow Belgian. . ............ 15c

$1 / 4$ I.b. LV.

$35 \mathrm{c} \quad \$ 1.00$

$35 \mathrm{c} \quad 1.00$

$35 \mathrm{c} \quad 1.00$

$35 \mathrm{c} \quad 1.00$

$35 \mathrm{c} \quad 1.00$

$35 \mathrm{c} \quad 1.00$

$35 \mathrm{c} \quad 1.25$

$25 \mathrm{c} \quad 90 \mathrm{c}$

$25 \mathrm{c} \quad 90 \mathrm{c}$ 
Henderson's True Early Snowball....... \$1/. ${ }^{1 / 2}$

Adams' Extra Select Dwarf Erfut. . . . . . . 1.50

Danish Giant or Dry Weather........... 1.50

\section{CELERY}

One-half Pound at Pound Price Oz. $\quad 1 / 4 \mathrm{Lb}$ Adams' Easy Blanching, Am. Grown 50c Paris Golden Self-Bleaching, Im-

ported................ 50c $\$ 1.25$

Lb. $\$ 4.00$

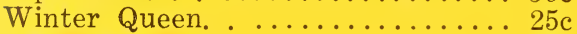

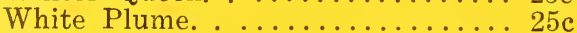

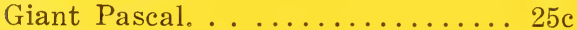

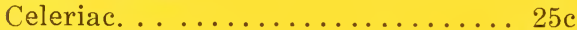

Golden Plume. . ..........\$1.50

1.75

6.00

$75 \mathrm{c}$

2.50

$75 \mathrm{c}$

$75 \mathrm{c}$

$75 \mathrm{c}$

2.50

2.50

5.00

2.00

\section{CUCUMBER}

Five Lbs. and Over 5 c Less Per Lb.

One-half Pound at Pound Price

The Adams'............... $50 \mathrm{c}$

Adams' Stays Green. .......... 20c

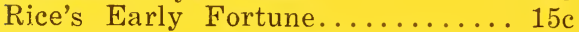

Improved Arlington White Spine... 15c

Extra Long or Evergreen White

Spine. . .................... 15c

Adams' Special. . . . . . . . . . . 20 c

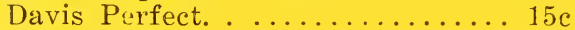

Short Green........................

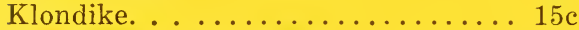

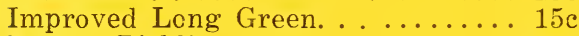

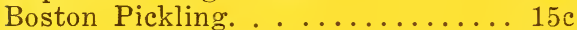

Adams' China-Long. ........ 50c

Gherkin or Burr. .......................

Hybrid White Spine......... 25c

$\begin{array}{cr}1 / 4 \mathrm{Lb} . & \text { Lb. } \\ \$ 1.50 & \$ 5.00 \\ 50 c & 1.75 \\ 35 c & 1.25 \\ 35 c & 1.25 \\ & \\ 35 c & 1.25 \\ 60 c & 2.00 \\ 40 c & 1.50 \\ 35 c & 1.25 \\ 35 c & 1.25 \\ 50 c & 1.50 \\ 35 c & 1.00 \\ \$ 1.50 & 5.50 \\ 40 c & 1.50 \\ 60 c & 2.00\end{array}$

\section{SWEET CORN}

25 Pounds at 100 Pound Price Lb. Adams' Dwarf, Extra Early. 25c Adams' Extra Early. . ... 25c Adams' Early. . ....... 25c Mammoth White Cory..... 35c

Early Evergreen. .......35c

Early Champion. ....... 35c

Country Gentleman. ..... 35c

Old Colony............ 35c

Mayflower............ 35c

Pocohontas. .......... 35c

Stowell's Evergreen. . ... 35c

Late Mammoth Sugar. . ... 35c

Golden Bantam........ 35c

Howling Mob. ......... 35c

Black Mexican. . . . . . . . 35c

Truckers' Favorite. ..... 30 c

White Pearl. ......... 30c
5 Lbs.

$\$ 1.15$

.90

.90

1.15

1.15

1.15

1.15

1.15

1.40

1.15

1.15

1.15

1.15

1.15

1.15

.75

.75

18.00

\section{EGG PLANT}

One-half Pound at Pound Price O

Improved New York.......... 50c

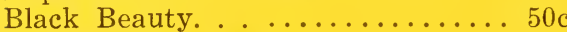

Large Round Purple. .......... 50c
10 Lbs. 100 Lbs.

$\$ 2.00 \$ \$ 15.00$

$\begin{array}{ll}1.50 & 12.00\end{array}$

$1.50 \quad 12.00$

$2.00 \quad 15.00$

$2.00 \quad 15.00$

$2.00 \quad 15.00$

$2.00 \quad 15.00$

$2.00 \quad 15.00$

$2.50 \quad 20.00$

$2.00 \quad 15.00$

$2.00 \quad 15.00$

$2.00 \quad 15.00$

$2.00 \quad 15.00$

$2.00 \quad 15.00$

$2.00 \quad 15.00$

$1.25 \quad 7.00$

$1.25 \quad 7.00$

\section{ENDIVE}

One-half Pound at Pound Price

Large Green Curled. ........... 15乞

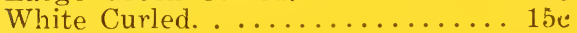

$1 / 1$ T.b.

L.b.

$\$ 1.50$

$\$ 5.00$

1.50

5.00

1.50

5.00

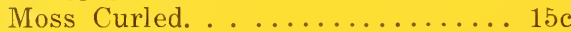

$1 / 4$ I.b.

$35 \mathrm{c}$

$35 \mathrm{c}$

Lb.

$35 \mathrm{c}$

$\$ 1.00$

1.00

Broad Leaf.

$15 \mathrm{c}$

$35 \mathrm{c}$

1.00 
One-half Pound at Pound Price Oz.

Philadelphia Dwarf German Green. 15c

Tall Curled Scotch. .......... 15c

Dwarf Green Curled Scotch........ 15c

\section{KOHL RABI}

One-half Pound at Pound Price Oz. Extra Early White Vienna...... 25c Early Purple Vienna. . ........ 25c

\section{LEEK}

One-half Pound at Pound Price

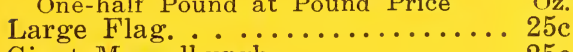

Giant Musselburgh. . .......... 25c

\section{LETTUCE}

Five Pounds and over 10c Less' per Pound

One-half Pound at Pound Price Oz.

Black Seeded Simpson......... 15c

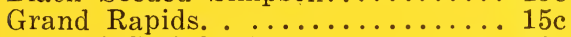

Adams' Satisfaction. ......... 20c

Early Curled Simpson. ......... 15c

New York, or Los Angeles....... 25c

Adams' Way a Head............15c

Prize Head. ............... 15c

Cos Lettuce, Romaine......... 20c

Unrivaled Summer. ............. 20c

Adams' Golden Forcing.......... 20c

May King. . .............. 20c

Improved Hanson. . ........... 15c

Iceberg. . ............... 20c

Denver Market. . . . . . . . . . . . 15c

Big Boston. .........................

Adams' Hot Weather................. 35c

$1 / 4 \mathrm{Lb}$.

$35 \mathrm{c}$

$35 \mathrm{c}$

Lb.

$35 \mathrm{c}$

1.00

1.00

$1 / 4 \mathrm{LW}$
$75 \mathrm{c}$

$75 \mathrm{c} \quad \$ 2.50$

$75 \mathrm{c} \quad 2.50$

$\begin{array}{rr}1 / 4 \text { Lb. } & \text { L.b. } \\ 75 \mathrm{c} & \$ 2.50 \\ 75 \mathrm{c} & 2.50\end{array}$

$\begin{array}{rr}1 / 4 \mathrm{Lb} . & \mathrm{Lb} . \\ 40 \mathrm{c} & \$ 1.25 \\ 40 \mathrm{c} & 1.25 \\ 50 \mathrm{c} & 1.50 \\ 40 \mathrm{c} & 1.25 \\ 75 \mathrm{c} & 2.50 \\ 40 \mathrm{c} & 1.25 \\ 40 \mathrm{c} & 1.25 \\ 50 \mathrm{c} & 1.50 \\ 50 \mathrm{c} & 1.50 \\ 50 \mathrm{c} & 1.50 \\ 50 \mathrm{c} & 1.50 \\ 40 \mathrm{c} & 1.25 \\ 50 \mathrm{c} & 1.50 \\ 40 \mathrm{c} & 1.25 \\ 40 \mathrm{c} & 1.25 \\ \$ 1.00 & 3.00\end{array}$

MUSKMELON OR CANTALOUPE

Five Pounds and Over 5 c Less Per Lb.

One-half Pound at Pound Price o

Hales' Best (new) .....................

Gold Lined Rocky Ford.........................

Extra Early Hackensack........ 15c

Pollock's No. 10-25............ 15c

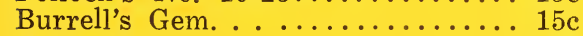

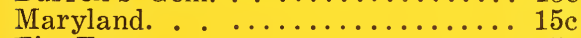

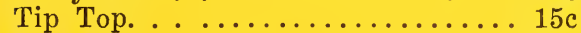

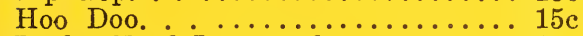

Rocky Ford Improved. ............. 15c

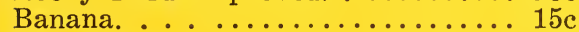

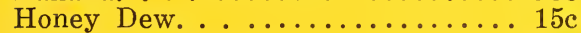

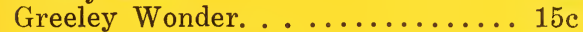

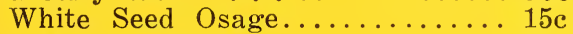

Lake Champlain. ............ 50 c

$\begin{array}{rr}1 / 4 \mathrm{Lb} . & \mathrm{Lb} . \\ 60 \mathrm{c} & \$ 2.00 \\ 40 \mathrm{c} & 1.25 \\ 40 \mathrm{c} & 1.25 \\ 40 \mathrm{c} & 1.25 \\ 40 \mathrm{c} & 1.25 \\ 50 \mathrm{c} & 1.50 \\ 50 \mathrm{c} & 1.50 \\ 50 \mathrm{c} & 1.50 \\ 40 \mathrm{c} & 1.25 \\ 50 \mathrm{c} & 1.50 \\ 50 \mathrm{c} & 1.75 \\ 60 \mathrm{c} & 2.00 \\ 40 \mathrm{c} & 1.50 \\ \$ 1.25 & 4.00\end{array}$

\section{WATERMELON}

Five Pounds and Over 5 c Less Per Lb.

One-half Pound at Pound Price Oz.

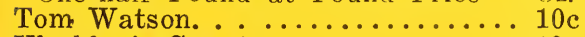

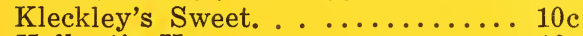

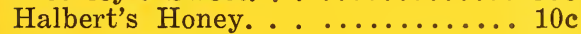

Georgia Rattlesnake. .........................

Irish Grey (New). . .........................

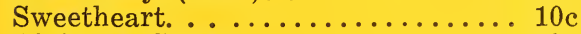

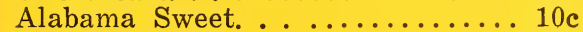

Florida Favorite. .......................

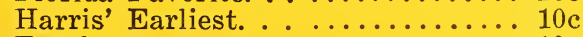

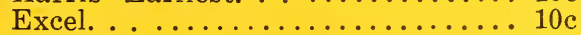

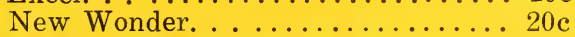

$\begin{array}{rr}1 / 4 \mathrm{Lb} . & \mathrm{Lb} . \\ 25 \mathrm{c} & 75 \mathrm{c} \\ 25 \mathrm{c} & 75 \mathrm{c} \\ 25 \mathrm{c} & 75 \mathrm{c} \\ 25 \mathrm{c} & 75 \mathrm{c} \\ 35 \mathrm{c} & \$ 1.00 \\ 25 \mathrm{c} & 75 \mathrm{c} \\ 25 \mathrm{c} & 75 \mathrm{c} \\ 25 \mathrm{c} & 75 \mathrm{c} \\ 25 \mathrm{c} & 75 \mathrm{c} \\ 25 \mathrm{c} & 75 \mathrm{c} \\ 40 \mathrm{c} & \$ 1.25\end{array}$


Five Pounds and Over 5 c Less Per Lb. One-half Pound at Pound Price Oz.

White London. ..............10c Black or Brown Mustard......... 10c

Southern Giant Curled. ......... 10c

Champion Moss Curled. ........ 10c

OKRA OR GUMBO

One-half Pound at Pound Price Oz.

White Velvet. .............10c

Perkins' Mammoth Long Pod...... 10c

Dwarf Green...............10c

\section{ONIONS}

Five Pounds at $10 \mathrm{c}$ per Pound Less

One-half Pound at Pound Price

Red Wethersfield. Oz.

Southport Large Red Globe....... 25c

Yellow Danvers Flat..........20c

Southport White Globe.......... 35c

White Portugal or Silverskin..... 30c

Yellow Globe Danvers.......... 25c

Mammoth Silver King.............. 30c

Riverside Sweet Spanish........ 40c

Australian Brown............ 20c

Prizetaker. . ............. 25c

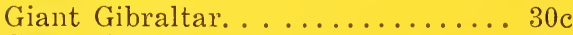

Green Bunch. ............. 25c

\section{PARSNIPS}

Five Pounds and Over $5 \mathrm{c}$ Less Per Lb.

One-half Pound at Pound Price

$\mathrm{Oz}$.

Hollow Crown Sugar. ..................

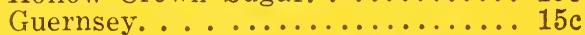

\section{PARSLEY}

One-half Pound at Pound Price Oz.

Double or Moss Curled.......... 15c

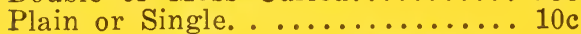

\section{PEPPER}

One-half Pound at Pound Price

Pimento. ........................ 50 c

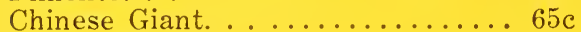

Ruby King. . . . . . . . . . . . . . . . . . . . .

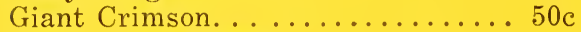

Bell or Bull Nose............. 50c

Ohio Crimson. ............ 50c

Neopolitan. ...................... 50c

Lorg Red Cayenne............ 50c

Livingston's Imp. Thick Long Red.. 60c

Royal King..............66 60

small Chili. . ...........6 60

World Beater or Goliath........60

Harris' Earliest. .......... \$1.00

\section{PEAS}

One-half Lb, at Lb. Price Ib.

Extra Early Pilot........ $35 \mathrm{c}$

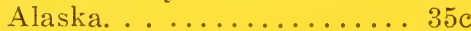

First and Best. ........... 35c

Gradus. . ..................... $35 \mathrm{c}$

Thos. Laxton. . ........ . 35c

Laxtonian. ............440

Premium Gem. ........ 35c

Bliss Everbearing. . ...... $35 \mathrm{c}$

Little Marvel. .......... 40

Improved Strategem. . .... 35

American Wonder. ...... 35c

Nott's Excelsior. ....... 35c

Sutten's Excelsior. . ..... 40

IIundred Fold. . .......... 40

$5 \mathrm{Lbs}$.

$\$ 1.40$

1.40

1.40

1.40

1.50

1.65

1.25

1.25

1.40

1.25

1.25

1.25

1.40

1.65

$1 / 4 \mathrm{Lb}$

$60 \mathrm{c}$

$75 \mathrm{c}$

$60 \mathrm{c}$

$\$ 1.00$

$90 \mathrm{c}$

$75 \mathrm{c}$

$90 \mathrm{c}$

1.50

$60 \mathrm{c}$

$75 \mathrm{c}$

$90 \mathrm{c}$

$75 \mathrm{c}$

Lb.

$\$ 2.00$

2.25

2.00

3.50

3.00

2.25

3.00

5.00

2.00

2.50

3.00

2.50

$1 / 4 \mathrm{Lb}$.

$25 \mathrm{c}$

$25 \mathrm{c}$

Lb.

$75 \mathrm{c}$

$75 \mathrm{c}$

1/4. Lb.

$35 \mathrm{c}$

$35 \mathrm{c}$

Lh.

$\$ 1.00$

1.00

$1 / 4 \mathrm{Lb}$.

Lb.

$\$ 1.50$

2.25

$\$ 5.00$

1.50

7.50

1.50

5.00

1.50

5.00

1.50

5.00

1.50

5.00

1.50

5.00

1.75

5.00

2.00

1.75

2.00

3.50

6.00

6.00

6.00

6.00

12.00

$10 \mathrm{Lbs} .100 \mathrm{Lbs}$.

$\$ 2.50 \$ 18.00$

$2.50 \quad 18.00$

$2.50 \quad 18.00$

$2.50 \quad 18.00$

$2.75 \quad 20.00$

$3.00 \quad 25.00$

$2.25 \quad 18.00$

$2.25 \quad 18.00$

$2.50 \quad 20.00$

$2.25 \quad 18.00$

$2.25 \quad 18.00$

$2.25 \quad 18.00$

$2.50 \quad 20.00$

$3.00 \quad 25.00$

25 LB. LOTS OF ANY ONE VARIETY OF PEAS, BEAN 
Telephone. ..........4 40c

\section{PUMPKIN}

Five Lbs. and Over 5 c Less Per I b.

One-half Pound at Pound Price

Large Cheese or Kentucky Field... 10c

Sweet Potato............. 15c

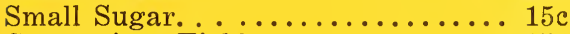

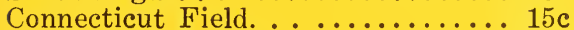

King of Mammoth............ 25c

Cushaw. ................. 15c

\section{RADISH}

Five Pounds and Over $5 \mathrm{c}$ Less Per Lb. One-half Pound at Pound Price

Adams' Scarlet Turnip Perfection

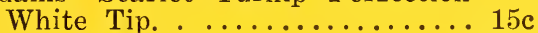

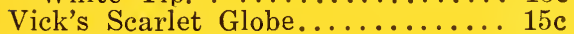

French Breakfast. ........... 15c

Sparkler White Tipped. ........ 15c

Philadelphia White Box. ........ 15c

Early White Turnip. ......... 15c

Wcod's Early Frame. ......... 15c

Long Scarlet Short Top........ 15c

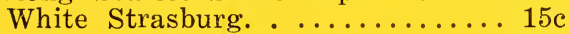

Early Long White Vienna....... 15c

White Lady Finger............ 15c

Improved Chartier. .......... 15c

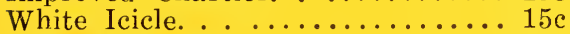

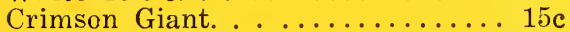

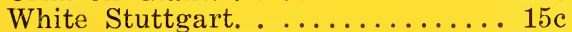

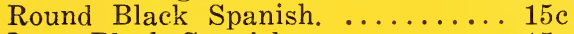

Long Black Spanish. ........... 15c

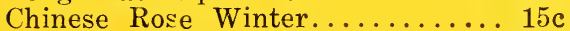

California White Mammoth....... 15c

Early Deep Scarlet Turnip....... 15c

Cincinnati Market. ............ 15c

\section{RHUBARB}

One-half Pound at Pound Price Oz.

Victoria Giant. . ......... 20c

Linneus. ................. 20c

$\begin{array}{rr}1 / 4 \mathrm{Lb} . & \mathrm{Lb} . \\ 20 \mathrm{c} & 50 \mathrm{c} \\ 35 \mathrm{c} & \$ 1.00 \\ 25 \mathrm{c} & 75 \mathrm{c} \\ 25 \mathrm{c} & 75 \mathrm{c} \\ 50 \mathrm{c} & 1.75 \\ 35 \mathrm{c} & 1.00\end{array}$

$1 / 4$ Lb. Lb.

$25 \mathrm{c} \quad 75 \mathrm{c}$

$35 \mathrm{c} \$ 1.00$

$25 \mathrm{c} \quad 75 \mathrm{c}$

$25 \mathrm{c} \quad 75 \mathrm{c}$

$25 \mathrm{c} \quad 75 \mathrm{c}$

$25 \mathrm{c} \quad 75 \mathrm{c}$

$25 \mathrm{c} \quad 75 \mathrm{c}$

$25 \mathrm{c} \quad 75 \mathrm{c}$

$25 \mathrm{c} \quad 75 \mathrm{c}$

$25 \mathrm{c} \quad 75 \mathrm{c}$

$25 \mathrm{c} \quad 75 \mathrm{c}$

$25 \mathrm{c} \quad 75 \mathrm{c}$

$25 \mathrm{c} 75 \mathrm{c}$

$25 \mathrm{c} \quad 75 \mathrm{c}$

$25 \mathrm{c} \quad 75 \mathrm{c}$

$25 \mathrm{c} \quad 75 \mathrm{c}$

$25 \mathrm{c} \quad 75 \mathrm{c}$

$25 \mathrm{c} \quad 75 \mathrm{c}$

$25 \mathrm{c} \quad 75 \mathrm{c}$

$25 \mathrm{c} \quad 75 \mathrm{c}$

25c $75 \mathrm{c}$

\section{SAGE}

One-half Pound at Pound Price

Large Leaved Garden.

SALSIFY OR OYSTER PLANT

One-half Pound at Pound Price

Mammoth Sandwich Island.......20

\section{SPINACH}

25 Pounds at 100 Pound Price Lb.

Bloomsdale or Savoy...... 30c

Princess Juliana. . ..... 40c

Viroflay Thick Leaf....... 30c

Long Standing. ......... 30c

King of Denmark........ 35c

New Zealand.

\section{SQUASH OR CYMBLING}

Five Lbs, and Over 5 c Less Per Lb.

One-half Pound at Pound Price Oz.

Mammoth White Bush. .......15c

Golden Summer Crookneck....... 15c

Chicago Warted Hubbard. ....... 15c

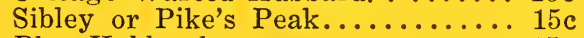

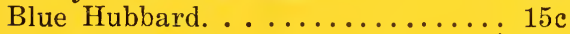

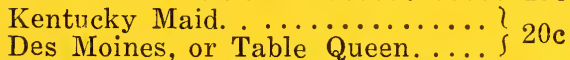

5 Lbs. 10 Lbs. $100 \mathrm{Jibs}$

$\$ 2.15 \quad \$ 18.00$

$\begin{array}{lll}1.90 & 3.50 & 28.00\end{array}$

$\begin{array}{ll}1.15-2.15 & 18.00\end{array}$

$2.15 \quad 18.00$

$2.75 \quad 25.00$

$1 / 4 \mathrm{Lb}$. Lb.

$35 \mathrm{c} \quad \$ 1.00$ 


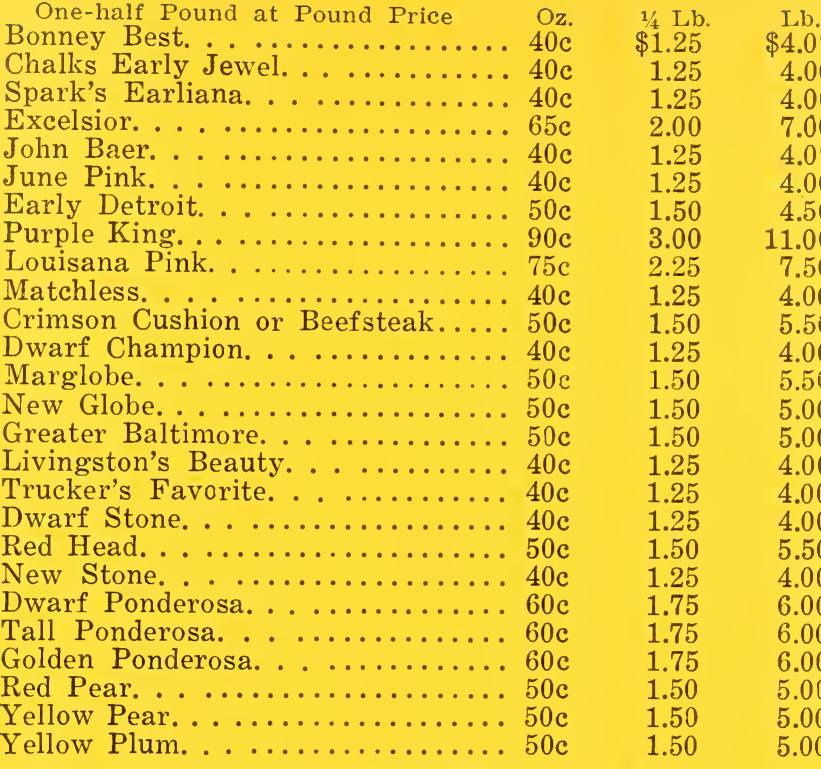

\section{TOBACCO}

Havana. ................. 50

Missouri Broad Leaf..........50

White Burley................50

Yellow Pryor............. 50 c

\section{TURNIPS}

Five Pounds and over $10 \mathrm{c}$ Less per Pound

One-half Pound at Pound Price Oz.

Purple Top Milan. ........... 15c

Early White Milan...........15c

Purple Top Globe. . .......... 10c

Purple Top Strap Leaf. ........ 10c

Snow Ball................. 15c

Rutabaga or Swede........... 15c

$\begin{array}{rr}1 / 4 \text { Lb. } & \text { Lb. } \\ 35 c & \$ 1.00 \\ 35 c & 1.00 \\ 20 c & 65 c \\ 20 c & 65 c \\ 35 c & 1.00 \\ 25 c & 75 c\end{array}$

\section{NORTHERN GROWN SEED POTATOES}

Red River Early Ohio, Early Rose, Early Triumph, Irish Cobblers, and other varieties. Prices furnished on application.

\section{FIELD SEEDS}

We carry a complete line of all kinds of field seeds, Red Clover, Alfalifa, Alsike Clover, Timothy, Sudan Grass, Blue Grass, Red Top, Cane, Millet, etc. Samples and prices furnished on application.

\section{SELECTED GRADED SEED CORN- Butted and Tipped}

Smoky Dent, New Mortgage Lifter, King of the Earliest. Trucker's Favorite, Reid Yellow Dent, White Pearl, Hickory King, and all standard varieties. Samples and prices furnished on application.

\section{OATS}

Kanota, Red Texas Rust Proof and White Bonanza. Samples and prices sent on application. 